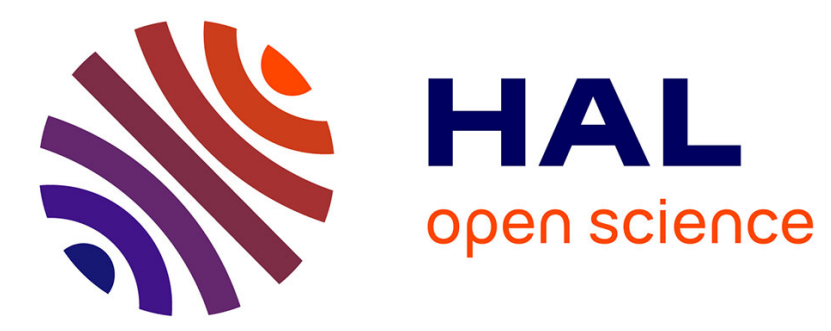

\title{
Bilan critique des opérations de repeuplement en petit gibier \\ D. Pépin
}

\section{To cite this version:}

D. Pépin. Bilan critique des opérations de repeuplement en petit gibier. Productions Animales, 1993, 6 (4), pp.269-275. hal-00896060

\section{HAL Id: hal-00896060 https://hal.science/hal-00896060}

Submitted on 1 Jan 1993

HAL is a multi-disciplinary open access archive for the deposit and dissemination of scientific research documents, whether they are published or not. The documents may come from teaching and research institutions in France or abroad, or from public or private research centers.
L'archive ouverte pluridisciplinaire HAL, est destinée au dépôt et à la diffusion de documents scientifiques de niveau recherche, publiés ou non, émanant des établissements d'enseignement et de recherche français ou étrangers, des laboratoires publics ou privés. 
INRA Prod. Anim., 1993, 6 (4), 269 - 275.
D. PÉPIN

INRA IRGM

BP 27, 31326 Castanet-Tolosan Cedex

\section{Bilan critique des opérations de repeuplement en petit gibier}

La raréfaction progressive du petit gibier a conduit à mettre en place des opérations pour reconstituer les populations naturelles ou augmenter leurs effectifs. Le bilan de ces opérations permet de dégager les conditions indispensables au repeuplement.

L'introduction en 1952 du virus de la myxomatose par le Dr Delille pour lutter contre les dégâts occasionnés en forêt par les lapins de garenne (Oryctolagus cuniculus) a entraîné presqu'aussitôt une chute de l'effectif de la plupart des populations de cette espèce. Devant l'ampleur de l'impact de ce virus, les chasseurs ont très vite envisagé d'introduire une espèce exotique de lapins résistante : leur choix s'est porté sur Sylvilagus floridanus et, malgré un avis défavorable du Comité Consultatif des Epizooties, plusieurs opérations de lâchers ont eu lieu dès 1953. Les dangers potentiels de ce type d'introduction en France furent alors immédiatement dénoncés par Giban (1954), notamment les risques

\section{Résumé}

S'appuyant sur une synthèse bibliographique des travaux disponibles, cet article établit un bilan critique des opérations de repeuplement menées en France à propos du Lapin de garenne, du Lièvre, de la Perdrix grise et de la Perdrix rouge. L'enchaînement des circonstances ayant entraîné la raréfaction progressive de ce petit gibier est tout d'abord très brièvement rappelé, le point de départ pouvant être attribué à l'introduction du virus de la myxomatose. Le recours à des lâchers comparatifs conduits par le service technique de l'Office National de la Chasse et la réalisation de quelques études plus ponctuelles par l'INRA permettent d'évaluer les chances de succès de ces tentatives de reconstitution ou de renforcement de populations naturelles dans divers contextes. Pour les lagomorphes, à côté de nombreux résultats chiffrés qui démontrent la forte variabilité dans le taux de survie des sujets introduits en fonction de facteurs variés (qualité de l'habitat d'accueil, époque du lâcher, aménagement des lieux du lâcher, statut des animaux), l'accent est mis sur la prise en compte nécessaire de la dynamique de leur comportement individuel et social. Pour les perdrix, on recommande d'une part d'entreprendre les opérations de repeuplement nécessaires sur de vastes unités géographiques pour qu'un nombre minimum de couples puisse s'implanter et se reproduire, et d'autre part d'utiliser uniquement des oiseaux d'origine locale sous peine de risquer une contamination génétique du stock naturel. Au vu de ces résultats, on souligne que la meilleure action en faveur du petit gibier reste une gestion raisonnée des populations naturelles. pathologiques pour la faune autochtone. Suite à une baisse de la virulence de la myxomatose et à une reconstitution progressive des populations de lapins, ces tentatives d'introduction s'estompèrent. Elles reprirent de façon clandestine au début des années 1970, par l'intermédiaire de lâchers d'individus provenant d'Italie. Pour une analyse plus détaillée concernant le statut de Sylvilagus floridanus en France, on consultera avec profit la mise au point publiée par Arthur et Chapuis (1983).

La disparition du lapin a entraîné en conséquence un report de la chasse sur d'autres espèces de petit gibier, notamment sur les lièvres (Lepus europaeus) et sur les perdrix grises (Perdix perdix) et rouges (Alectoris rufa). En plus de la transformation et de la dégradation des habitats, ce phénomène de transfert a contribué à une raréfaction progressive du petit gibier. L'attitude commune des responsables cynégétiques a alors été de se tourner vers des opérations de lâchers d'animaux de diverses origines (gibier d'importation ou issu d'élevages), sans, le plus souvent, envisager une limitation dans les prélèvements (figure 1).

Devant l'échec d'une telle politique, et bien que la pression de chasse ne puisse être incriminée comme la seule responsable du phénomène de régression (Havet et Biadi 1990), une démarche nouvelle consiste à combiner diverses mesures pour favoriser le redémarrage des populations à trop faible effectif : elles allient le plus souvent l'arrêt temporaire de l'exercice de la chasse, l'aménagement du territoire (cultures à gibier, implantation de haies), l'adoption de règles de protection pour réduire les pertes accidentelles (utilisation de 
procédés d'effarouchement, conduite des récoltes du centre des parcelles vers la périphérie plutôt que d'adopter le processus inverse) et la mise en place de véritables opérations de repeuplement. Dans ces conditions, l'objectif visé est que les sujets lâchés fassent souche,

Figure 1. Enchaînement de circonstances ayant entraîné la raréfaction progressive du petit gibier.

Introduction du virus de la myxomatose

Déclin des populations de lapins

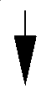

Report de l'effort de chasse sur d'autres espèces de petit gibier (Lièvre, Perdrix grise, Perdrix rouge)

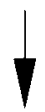

Raréfaction progressive du petit gibier

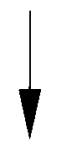

Opérations de lâchers d'espèces autochtones et exotiques (dont Sylvilagus floridanus)

et développement des élevages en captivité étroite
La réussite des opérations de repeuplement dépend dans une large mesure de la qualité du milieu d'accueil vis à vis des sujets lâchés. Pour le Lapin de garenne, une meilleure survie est constatée par des lâchers pratiqués en milieu ouvert (champs de céréales) par rapport à des opérations conduites dans des habitats plus fermés (bois, bosquets, broussailles, landes).

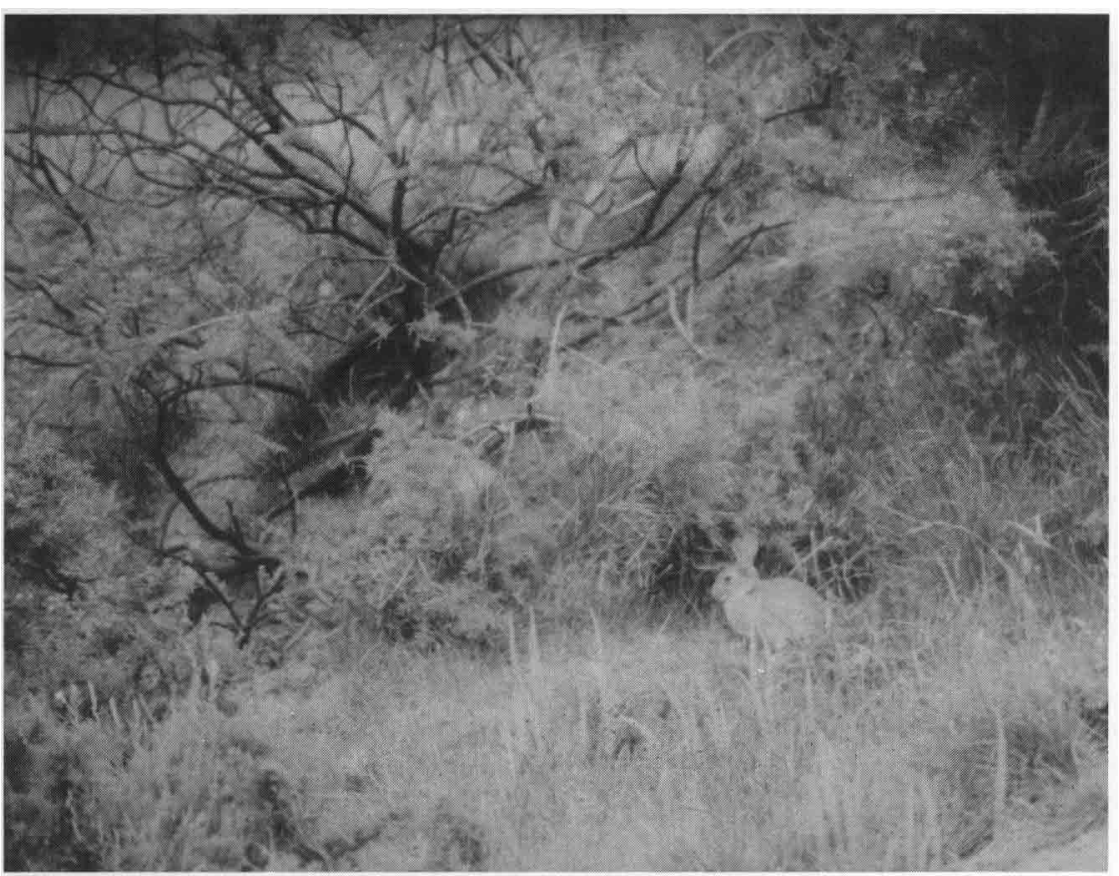

autrement dit qu'ils se reproduisent. Les lâchers sont donc destinés à permettre à la population de revenir à un niveau compatible avec son renouvellement annuel automatique. Cet article dresse un bilan critique de diverses opérations de repeuplement menées en France, qui ont concerné le Lapin de garenne, le Lièvre, la Perdrix grise et la Perdrix rouge. Il complète la synthèse de Havet et Biadi (1990) consacrée uniquement aux travaux menés par l'Office National de la Chasse sur ces espèces de petit gibier, ainsi que sur le Faisan (Phasianus colchicus) et sur le Colvert (Anas platyrhynchos).

\section{1 / Le Lapin de garenne}

Le recours à des lâchers comparatifs de différents lots d'animaux a permis au service technique de l'Office National de la Chasse d'évaluer l'influence respective de différentes variables sur le succès des opérations de repeuplement hivernal en lapins de garenne. Une série d'expérimentations portant sur près de 2400 sujets marqués a d'abord permis de constater que, dans tous les cas de figure, la mortalité survient de façon très rapide, surtout dans les 2 semaines qui suivent le lâcher. La survie globale constatée 21 jours après le lâcher $(J+21)$ s'avère en moyenne supérieure dans le cadre d'opérations réalisées dans des secteurs où restent encore quelques lapins autochtones que dans celles où il n'en subsiste plus ( $35 \%$ vs $20 \%$ ). A J+90, la survie moyenne est de l'ordre de $29 \%$ dans le premier cas, contre $16 \%$ dans le second cas (voir figure 2). La survie des animaux est d'autre part supérieure en milieux ouverts (céréales, pâtures) par rapport aux milieux fermés (bois, bosquets, haies, landes, broussailles) (soit $25 \%$ vs $19 \%$ ) ; elle s'avère également plus importante en présence de céréales $(34 \%)$ par rapport à des zones en pâture (19\%, voir figure 2$)$. En définitive, ce sont les lapins lâchés en milieux ouverts et en présence de céréales qui ont le meilleur taux de survie ( $43 \%$ ), alors que ceux lâchés en milieux fermés et dans des zones en pâture ont les plus faibles chances de survivre (environ $15 \%$ seulement, voir figure 2). Si la présence de sujets autochtones au moment du lâcher n'a pas d'effet significatif sur le taux de survie des lapins lâchés en milieux fermés, par contre elle apparaît comme bénéfique dans le cadre d'opérations conduites en milieux ouverts $(34 \%$ contre seulement $14 \%)$. Grâce à des lâchers de lots de 10 à 20 individus, il a pu être remarqué une très forte variabilité dans la survie à J+90 entre lots : au sein d'une même opération, elle peut intéresser deux tiers des sujets lâchés ou être nulle.

Une grande majorité $(95 \%)$ des animaux revus ou retrouvés morts sont le plus souvent découverts dans un cercle de $500 \mathrm{~m}$ de rayon autour du point de lâcher. Leur dispersion est très rapide puisqu'ils sont stabilisés dès la 3ème ou 4ème semaine suivant le lâcher. En conséquence, il ne semble pas légitime d'incriminer une dispersion à longue distance pour expliquer le faible taux de survie constatée à 
l'échelle locale. Grâce à des contrôles réalisés sur le premier mois du lâcher, il a pu être constaté une perte de poids importante des sujets lâchés (de 20 à $30 \%$ en moyenne), surtout entre $\mathrm{J}+10$ et $\mathrm{J}+20$. Néanmoins, une fois ce cap franchi, les animaux "récupèrent" assez rapidement puisque le poids enregistré à $J+30$ dépasse celui à la date du lâcher. La prédation et les problèmes pathologiques sont les principales causes de mortalité (60\% pour ces 2 facteurs).

En se basant sur le fait que l'organisation sociale du Lapin de garenne est très tributaire de l'utilisation collective de terriers ou d'abris naturels, il a paru logique de réaliser des aménagements artificiels destinés d'une part à améliorer la survie des sujets lâchés, et d'autre part à limiter leur dispersion (Mauvy et al 1991). Au sein de 2 zones du Massif Central, il a donc été procédé à la comparaison de résultats obtenus à partir de points de lâcher aménagés et non aménagés (servant de témoins). La cohésion des groupes a été estimée par observations directes, et la fréquentation des divers aménagements réalisés a été quantifiée en tenant compte des signes de présence du lapin autour de chaque aménagement. Il a d'abord été mis en évidence un cycle annuel dans la fréquentation des aménagements, caractérisé par une utilisation forte en hiver et faible en été. Si la cohésion des groupes, la dispersion, et la survie des ani-

Figure 2. Influence de diverses variables sur la survie des lapins de garenne lors de repeuplements hivernaux (nombre de survivants à $J+90$ par rapport au nombre de lâchés), modifié d'après Arthur (1989).

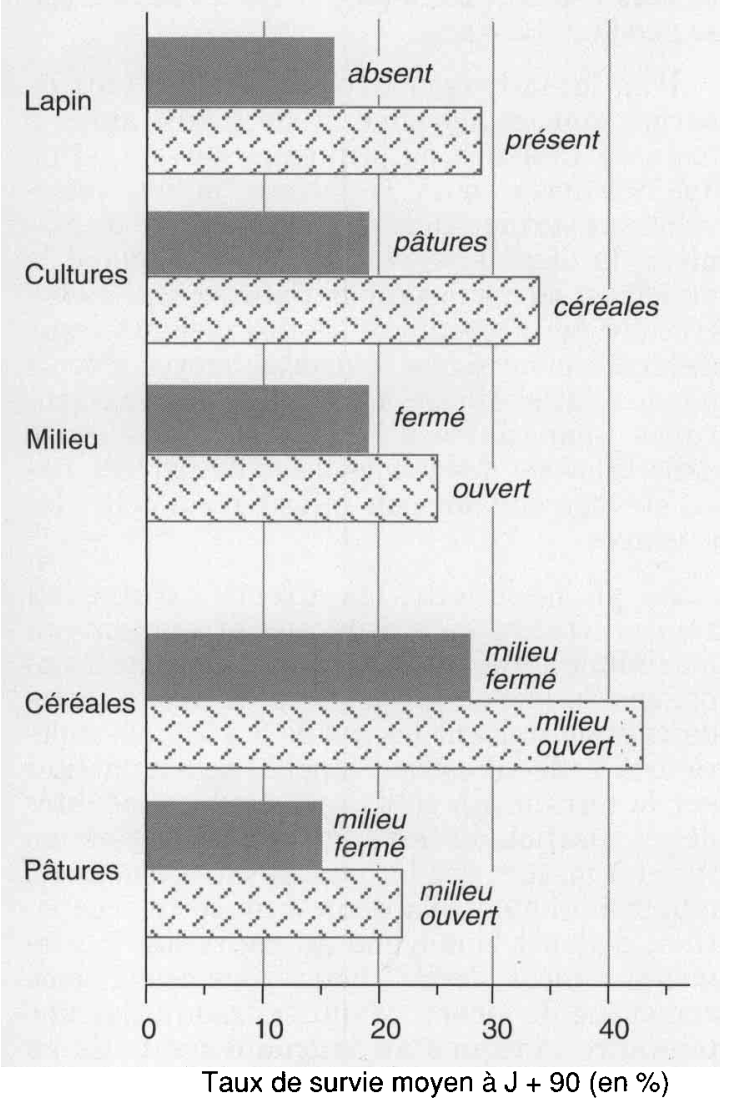

maux lâchés se trouvent améliorées au départ (dans la semaine qui suit le lâcher), ces avantages initiaux ne se sont pas révélés durables. D'après les auteurs de cette étude, il semble que ce soit l'absence de véritables liens sociaux entre la plupart des animaux lâchés qui explique ces échecs. En conséquence, ils recommandent de prendre davantage en compte ces liens sociaux dans les opérations de repeuplement, en cherchant par exemple à respecter les conditions de leur installation et de leur maintien au moment du lâcher des animaux.

Le devenir à plus long terme des populations repeuplées dépend dans une large mesure du succès de la reproduction des animaux qui ont réussi à s'implanter (Arthur 1989). Il semble nécessaire qu'une densité minimale puisse être obtenue à J+90. Encore faut-il qu'elle soit complétée par un recrutement suffisant de jeunes lapereaux, c'est-à-dire par un taux de survie conséquent. Il est constaté en effet que la reproduction de la première année compense à peine les pertes du lâcher et qu'il faut donc attendre au mieux la deuxième année pour que la population secourue commence à se développer.

\section{2 / Le Lièvre}

Les lâchers de lièvres ont d'abord été pratiqués en France à partir d'animaux importés des pays d'Europe Centrale. Jusqu'à la fin des années 1970 , de 150 à 200000 sujets en moyenne ont été annuellement introduits, les problèmes posés par ce type de repeuplement étant d'ailleurs explicités dès cette époque par Havet (1975) : cet auteur distingue quatre aspects principaux dans la pathologie de ces lièvres (d'origine traumatique, infectieuse, parasitaire et liée aux phénomènes d'agression), auxquels viennent s'ajouter des problèmes d'ordre éco-éthologiques (relation avec la population locale, sensibilité aux prédateurs très accrue, grand déplacement des animaux avant l'adoption d'un cantonnement définitif). Parallèlement, un engouement pour les élevages de lièvres s'est progressivement développé, la production étant estimée annuellement à 150000 levrauts environ depuis la fin des mêmes années 1970 . C'est dans ce contexte que l'Office National de la Chasse a entrepris d'évaluer la survie et le comportement de lièvres lâchés en nature en mettant progressivement en place dans divers sites expérimentaux des contrôles qui ont surtout porté sur des levrauts âgés de 1 à 3 mois (environ 2500 animaux), mais aussi sur quelques 700 individus naturels importés.

L'estimation du taux de survie des lièvres lâchés se base la plupart du temps sur les reprises à la saison de chasse suivante (Fiechter et al 1978, Biadi et Benmergui 1989). Pour les levrauts introduits au printemps ou en été, le taux de reprise varie globalement de 4 à $20 \%$, selon les années et les sites d'étude. D'après Fiechter et al (1988), le passage en parc de pré-lâcher situé sur le lieu 
même du lâcher n'améliore pas de façon sensible le taux de reprise. Si l'utilisation de levrauts élevés sur litière et sur herbe permet d'obtenir un meilleur résultat par rapport à des sujets élevés sur grillage, on constate néanmoins que leur taux de reprise reste toujours relativement faible, de l'ordre de $20 \%$. En comparant le devenir de levrauts issus d'élevage et de levrauts naturels équipés d'émetteur, il a été constaté que leur taux de survie est de l'ordre de $21 \%$ pour les premiers contre $44 \%$ pour les seconds 2 mois après le lâcher et de 15 contre $38 \% 5$ mois après le lâcher (Biadi et Benmergui 1989). Le cap des 3 premiers jours qui suivent la date du lâcher est particulièrement difficile à franchir pour les levrauts nés en élevage, le stress subi par ces animaux jouant alors un rôle important (Marboutin et al 1990). Pour les lièvres naturels importés, les contrôles réalisés se déroulent en règle générale à l'automne suivant, soit 9 à 10 mois après la date du lâcher. Leur taux de reprise moyen oscille entre 13 et $30 \%$ suivant les années et les territoires (Biadi et Benmergui 1989). Le suivi d'opérations de repeuplement réalisées dans l'est de la France en décembre ou en janvier, a permis d'une part de constater qu'il est préférable d'effectuer les lâchers au début de l'hiver, d'autre part de montrer que le passage en parc de prélâcher n'a aucune incidence bénéfique sur la dispersion des sujets lâchés en plaine (Benmergui et al 1990). Ce suivi a également permis de préciser que si la distance moyenne de reprise de ce type de lièvres avoisine les $2 \mathrm{~km}$ en plaine, elle est significativement plus faible dans le cas de lâchers ayant lieu en zone de côteaux que dans le cas de lâchers réalisés en moyenne montagne.

Pour les levrauts d'élevage qui réussissent à s'implanter, l'adoption d'un secteur de cantonnement définitif est précédée par une phase de dispersion de faible amplitude qui s'accompagne de changements fréquents de gîtes de jour et, le cas échéant, par un pré-cantonnement dans une zone restreinte (Pépin et Cargnelutti 1985, 1987). Durant toute cette période d'apprentissage, les levrauts ont un rythme d'activité variable mais, en règle générale, ils commencent à se déplacer bien avant le coucher du soleil et se gitent dès son lever. Après cantonnement, leur niveau d'activité locomotrice est plus réduit, et ce aussi bien de jour que de nuit. On assiste également à la mise en place d'une structuration progressive de leur espace individuel qui se caractérise notamment par la constitution d'un véritable réseau de gîtes et par la séparation entre zone d'alimentation (gagnage) utilisée durant la nuit, et gîte de jour, soit de dépar! se d'arrivée. En cas de dérangements (travaux agricoles le plus souvent), les levrauts cantonnés peuvent réutiliser momentanément des secteurs qu'ils ont eu l'occasion d'explorer lors de leur phase de dispersion ou de int-iantonnement.

Le fait pour un adulte sauvage d'être libéré dans un milieu inconnu lui confère le statut d'individu "naïf". Par conséquent, on assiste globalement aux mêmes étapes que pour les levrauts d'élevage, à savoir d'abord essaimage autour du point de lâcher avec premiers gîtes éloignés les uns des autres, puis phase d'insertion avec concentration des gîtes dans une zone restreinte (Ricci 1983, Pépin et Cargnelutti 1985).

\section{3 / La Perdrix grise et la Perdrix rouge}

Comme dans de nombreux autres pays européens, la régression des populations des 2 espèces de Perdrix s'est amorcée en France à partir des années 1970 . Pour juguler ce déclin, des lâchers d'oiseaux d'élevage ont alors été réalisés à petite échelle, la période la plus favorable semblant se situer en août, avec des oiseaux âgés au minimum de 7 à 8 semaines (Birkan 1977). Mais, avec un certain recul, il est apparu que l'efficacité à moyen terme de telles opérations de repeuplement est très limitée.

A l'heure actuelle, on s'oriente donc davantage vers des tentatives de reconstitution (là où il ne subsiste plus de sujets sauvages) ou de renforcement de populations naturelles (là où la densité est très faible) sur des zones de plusieurs milliers ou dizaines de milliers d'hectares (Biadi 1989). Diverses opérations expérimentales de repeuplement en perdrix ont été réalisées et contrôlées par l'Office National de la Chasse dans 5 régions, pour un effectif total d'environ 70000 oiseaux lâchés. Pour leur organisation, plusieurs mesures ont été prises, notamment l'aménagement de sites de lâcher grâce à la mise en place de parcs avec postes d'agrainage et de points d'eau, la répétition des lâchers pendant 2 à 3 ans, et l'arrêt de la chasse pendant 3 à 5 ans.

Pour les lâchers effectués en été, les taux de survie sont en moyenne de 50 à $70 \%$ après 2 mois, de 15 à $25 \%$ au printemps suivant, et de 5 à $10 \%$ après un an. Le facteur "milieu" interviendrait surtout au niveau de la survie printanière, la dispersion étant plus forte quand le milieu est moins favorable (Catusse et al 1988). L'étude de la productivité des oiseaux issus d'élevage montre que le nombre moyen d'oeufs pondus est similaire à celui des oiseaux sauvages ; par contre il semble que la réussite dans l'élevage des jeunes issus de parents nés en élevage soit un peu inférieure à celle des sauvages.

Le plus souvent, les effectifs d'oiseaux reproducteurs au printemps atteignent un maximum après une seule répétition des campagnes de lâchers, le nombre de sujets introduits étant suivant les cas de 2 à 20 fois supérieur à l'effectif estimé capable de s'implanter sur le terrain. En se basant sur les modalités de colonisation du territoire par les oiseaux au fur et à mesure des lâchers, Brun et Aubineau (1989) indiquent d'ailleurs que cette occupation, d'abord homogène au cours des 2 premières années, devient hétérogène dès la 3ème campagne de lâcher, ce qui se traduit par une tendance marquée au regroupement. Ils en concluent que la saturation du milieu ainsi 
mise en évidence peut expliquer les échecs rencontrés dans les tentatives de repeuplement réalisées sur des zones de trop petite taille.

Après l'arrêt des lâchers, le devenir des populations varie d'une région à l'autre. Les opérations conduites dans le Bassin Parisien (renforcement de population) et dans l'Ouest de la France (reconstitution de population) ont été couronnées de succès puisqu'elles ont permis par la suite la reprise de la pratique de la chasse. Dans le Massif Central, les opérations réalisées dans les milieux les moins défavorables ont réussi. Par contre, celles tentées dans des secteurs peu accueillants ont échoué (cas de la plupart des essais tentés dans le Sud-Ouest et dans les Hautes-Alpes). La synthèse de ces résultats semble indiquer que la juxtaposition de secteurs plus ou moins favorables aux perdrix est un argument en faveur d'actions de repeuplement à entreprendre sur des unités géographiques suffisamment vastes pour permettre à un nombre minimum de couples de s'implanter et de se reproduire, en tenant compte des aléas climatiques (plus d'une cinquantaine de couples pour la perdrix rouge, d'après l'étude de Catusse et al 1988).

Parmi les facteurs susceptibles d'expliquer l'échec des expériences de lâchers de perdix grise menées dans les Hautes-Alpes, Léonard (1988) signale que, dans le cadre de ces essais, aucun spécimen véritablement adapté au milieu montagnard n'a pu être disponible. En conséquence, cet auteur recommande de conserver la souche naturelle lorsqu'elle subsiste encore et d'aménager le territoire, plutôt que d'essayer d'implanter des oiseaux non adaptés. Cette spécificité des souches locales à protéger est également la raison invoquée par Lescourret (1988) pour critiquer les introductions de perdrix grises d'élevage dans les Pyrénées, l'hypothèse d'une contamination génétique du stock naturel dans les Pyrénées Orientales étant d'ailleurs avancée par Blanc et al (1987).
Au cours de l'été, la récolte des cultures peut provoquer une mortalité importante des perdrix qui couvent leur nid. La récupération des oeufs, leur couvaison puis l'élevage des perdreaux (ici des perdreaux gris) par des poules naines est un moyen qui permet de limiter l'impact de ce type de pertes.

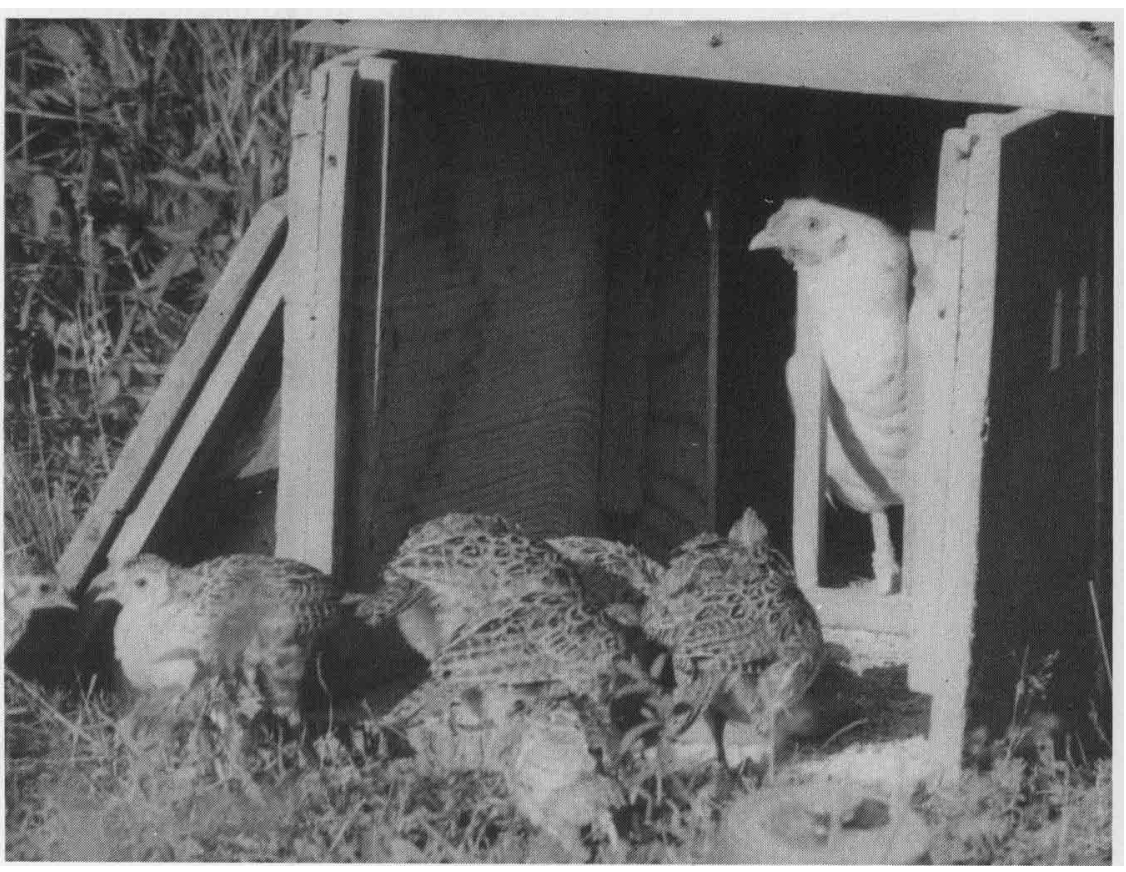

Figure 3. Fluctuation pluri-annuelle d'une population de lièvres après une forte mortalité accidentelle, dans trois situations différentes.

Densité de population

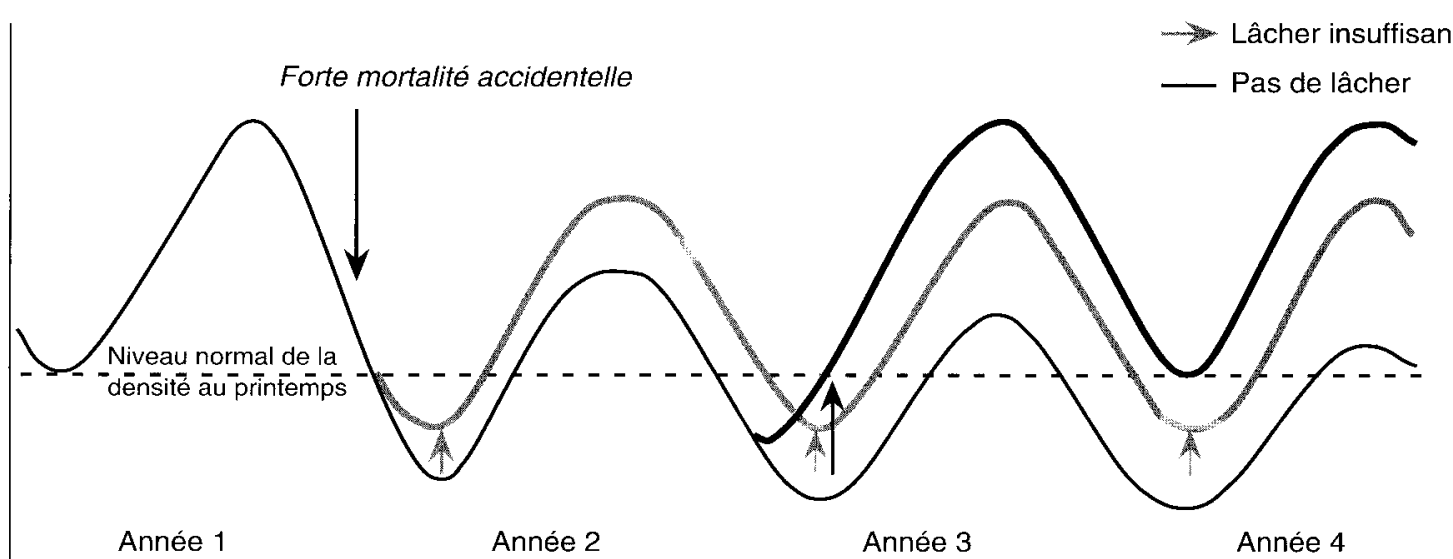

A des échelles géographiques très limitées, il existe également chez la Perdix rouge adulte des variations significatives dans le format corporel (Pépin 1988) et dans le polymorphisme génétique (Blanc 1988) pour des sujets collectés à la chasse. Il n'est donc pas exclu que le recours à des oiseaux d'élevage non adaptés d'une part explique le faible succès des opérations de repeuplement, et d'autre part contribue à jouer un rôle non négligeable au plan génétique.

\begin{tabular}{|c|c|c|c|c|c|c|c|}
\hline $\begin{array}{c} \\
\text { Printemps }\end{array}$ & $\begin{array}{c}1 \\
\text { Automne }\end{array}$ & \begin{tabular}{c|c} 
\\
Printemps
\end{tabular} & $\begin{array}{c}1 \\
\text { Automne }\end{array}$ & \begin{tabular}{c|} 
\\
Printemps
\end{tabular} & \begin{tabular}{l|l} 
& \\
Automne
\end{tabular} & \begin{tabular}{l|l} 
& \\
Printemps
\end{tabular} & $\begin{array}{l}1 \\
\text { Automne }\end{array}$ \\
\hline
\end{tabular}




\section{Conclusion}

Si la prédation apparaît toujours comme le facteur principal incriminé pour expliquer la mortalité importante des animaux lâchés, il est vraisemblable que, dans un certain nombre de cas, le rôle direct réel qui lui est attribué occulte d'autres paramètres plus difficiles à mettre en évidence (stress, mauvais état général, etc).

D'un point de vue appliqué, l'ensemble des études que nous venons très succinctement de résumer semble indiquer que lorsqu'il est vraiment urgent de pratiquer des opérations de repeuplement de petit gibier, il est alors tout à fait indispensable de les organiser sur de vastes zones, avec des animaux de qualité. Les raisons qui ont conduit au déclin des populations secourues doivent être très soigneusement analysées et reconnues comme telles par le plus grand nombre. Si la pression de chasse est en cause, elle doit être impérativement suspendue pendant plusieurs années. Si la dégradation du milieu est en cause, la mise en place et l'entretien d'aménagements appropriés à l'espèce en péril s'imposent avant toute tentative d'introduction. Compte tenu des taux élevés de mortalité et de la capacité de dispersion des sujets survivants, il demeure enfin indispensable de réaliser les lâchers avec un nombre très conséquent d'individus marqués (voir figure 3).

En se basant uniquement sur le prix d'achat des lièvres à lâcher, Biadi et Benmergui (1989) estiment qu'il faut, suivant les cas, dépenser de 6000 à $20000 \mathrm{~F}$ pour espérer obtenir 2 reproducteurs supplémentaires par 100 hectares. En conséquence, ils recommandent de privilégier la gestion des populations naturelles afin de permettre leur accroissement sans avoir recours au repeuplement systématique.

\section{Références bibliographiques}

Arthur C., 1989. Les opérations de repeuplement hivernal en lapins de garenne. Bulletin mensuel O.N.C., $139,15-28$

Arthur C., Chapuis J.L., 1983. L'introduction de Sylvilagus floridanus en France : Historique, dangers et expérimentation en cours. Compte Rendu de la Société de Biogéographie, 59, 333-356.

Benmergui M., Reitz F., Fiechter A., 1990. Taux de reprise et dispersion de lièvres (Lepus europaeus) sauvages d'Europe Centrale relâchés dans l'est de la France. Gibier Faune Sauvage 7, 255-274

Biadi F., 1989. Reconstitution ou renforcement de populations naturelles de perdrix. Bulletin mensuel O.N.C., 136, 5-12.

Biadi F., Benmergui M., 1989. Repeuplements en lièvres : survie et dispersion des lièvres et des levrauts lâchés. Bull. mensuel O.N.C., 134, 26-31.

Birkan M., 1977. Lâchers de perdrix grise d'élevage (Perdix perdix), valeur pour le repeuplement. Bull. mensuel O.N.C., No Sp. Scient. Techn., nov. 1977 , $47-122$

Blanc F., 1988. Variabilité génétique chez la Perdrix rouge (Alectoris rufa) (Phasianidae). Convention 83101, Univ. P.-Valéry - Secrétariat auprès du Premier Ministre chargé de l'Environnement et de la Qualité de la vie, $32 \mathrm{p}$.

Blanc F., Ledème P., Blanc Ch., 1987. Quelques résultats des travaux menés su! ': Bbilité génétique chez la perdrix grise. Bulietin mensuel O.N.C., 113, 11-13.

Brun J.C., Aubineau J., 1989. La reconstitution des populations de perdrix rouges (Alectnris rufa) et grises (Perdix perdix) à l'aide d'oises: $\mathrm{x}$ d cievage. Gibier Faune Sauvage 6, 205-223.

Catusse M., Goudenèche D., Biadi F., Marchandeau S., 1988. Repeuplement en perdrix rouges (Alectoris rufa) dans le sud-ouest de la France. Bulletin mensuel O.N.C., 126, 5-16.
Fiechter A., Havet P., Fournier J.Y., Brouillard A., 1978. Expériences et résultats de lâcher de levrauts d'élevage tirés à Vendenesse-sur-Arroux. Bull. mensuel O.N.C., $N^{\circ}$ Sp. Scient. Techn., nov. 1978, 83-113.

Fiechter A., Benmergui M., Marchandeau S., Scherrer B., Francois A., Labous Y., 1988. Influence du mode de lâcher et du mode d'élevage sur la réussite d'opérations de lâcher de levrauts (Lepus europaeus). Gibier Faune Sauvage 5, 1-14.

Giban J., 1954. Notes sur les dangers de l'introduction en France de léporidés américains. Bulletin Officiel du Conseil Supérieur de la Chasse, 36-39.

Havet P., 1975. Contribution à l'étude des problèmes posés par les repeuplements en lièvres d'importation. Bull. mensuel O.N.C., $N^{\circ}$ Sp. Scient. Techn., 1975-4, 13-67.

Havet P., Biadi F., 1990. Réintroductions et soutiens de populations d'espèces de petit gibier. Revue d'Ecologie (Terre Vie), Suppl. 5, 261-289.

Léonard P., 1988. Bilan des expériences de lâchers de perdrix grise effectuées par l'Office National de la Chasse dans le département des Hautes-Alpes. Bulletin mensuel O.N.C., 129, 9-15.

Lescourret F., 1988. Eléments de répartition de la perdix grise (Perdix perdix hispaniensis Reich.) dans les Pyrénées françaises. Gibier Faune Sauvage 5, 123-148.

Marboutin E., Benmergui M., Pradel R., Fiechter A. 1990. Survival patterns in wild and captive-reared leverets (Lepus europaeus Pallas) determined by telemetry. Gibier Faune Sauvage 7, 325-342.

Máury B., Peroux R., Lartiges A., Sidaine M., 1991. Repeuplement en lapins de garenne : résultats des essais effectués dans le nord du Massif Central. Deuxième et dernière partie : la fréquentation des aménagements et la recherche de leur influence sur le devenir des animaux lâchés. Bulletin mensuel O.N.C., 158, 13-20. 
Pépin D., Cargnelutti B., 1985. Dispersion et cantonnement de lièvres de repeuplement (Lepus europaeus). Biology of Behaviour 10, 353-365.

Pépin D., Cargnelutti B., 1987. Développement de stratégies d'utilisation de l'espace et du temps lors de l'implantation en nature de levrauts (Lepus europaeus) issus d'élevage. Gibier Faune Sauvage 4, 149-164.
Pépin D., Cargnelutti B., Mathon J.F., 1988. Variation géographique de la taille chez la Perdrix rouge (Alectoris rufa). Gibier Faune Sauvage 5, 213220 .

Ricci J.C., 1983. Suivi d'un lâcher de lièvres d'importation (Lepus europaeus Pallas) au moyen de la radiotélémétrie : mortalité, dispersion et utilisation de l'espace. Acta Oecol., Oecol. Applic., 4, 31-46.

\section{Summary}

\section{Critical review of small game restocking schemes}

Based on a synopsis of available literature data, this article makes a critical review of the Wild Rabbit, Brown Hare, Grey Partridge and Red Partridge restocking operations carried out in France. A series of circumstances and conditions which gradually reduced the stocks are briefly reminded, the starting point possibly being the introduction of the myxomatosis virus. Comparative releases performed by the Office National de la Chasse (French National Game and Hunting Agency) and a few more isolated studies conducted by INRA make it possible to assess the success rate of these attempts to restore or reinforce natural populations in various contexts.
With regard to lagomorpha, beside a number of quantitative data showing the highly variable survival rate of the subjects introduced according to various factors (quality of habitat, time of release, release location setting, animal condition), the emphasis is put on the need to take their social and individual behaviour into account. For partridges, it is recommended that the necessary restocking operations be undertaken in wide geographical areas, so that a sufficient number of pairs can settle and reproduce, and that only local birds be used, to prevent genetic contamination of the natural stock.

PÉPIN D., 1993. Bilan critique des opérations de repeuplement en petit gibier. INRA Prod. Anim., 6 (4), 269 275. 